\title{
Process analysis and optimization of Nd:YAG continuous laser grooving for fracture splitting of a C70S6 connecting rod
}

\author{
Kou Shuqing ${ }^{1,}$, Gao Yan ${ }^{1, b}$ and Lin Baojun ${ }^{1, c}$ \\ ${ }^{1}$ Roll Forging Research Institute, Jilin University, Changchun, China, 130022 \\ akousq@jlu.edu.cn, bgaoyan_ly@126.com, ${ }^{\mathrm{C}}$ linbj@jlu.edu.cn
}

\section{Keywords: Continuous laser; Grooving; Notch; Fracture splitting; Process optimization}

Abstract. Fracture splitting processing, which has board prospects in the precision manufacturing of engine connecting rod, has been widely applied in production so far, and laser pre-processing a fracture splitting notch which is a notch as the fracture initiation source for the subsequent splitting process is the important link in the fracture splitting technology. Therefore, the continuous laser grooving for fracture splitting was analyzed and the influence parameters during the continuous laser grooving were studied in this article. Based on the physical process between the laser beam and materials during laser grooving, the finite element analysis model was built for the continuous laser grooving, 3D gauss conical heat source model was checked based on the relative experimental investigation, then the numerical simulation of temperature field of continuous laser grooving of steel C70S6 was carried out, and the influence rule of main process parameters on the morphology of the fracture splitting notch was obtained. Research results showed that the dimension of the notch processed by continuous laser met the production requirement; notch depth and notch width was positively correlated with laser output power but negatively correlated with transverse speed; facula radius was negatively correlated with notch depth but positively correlated with notch width; it is not recommended that transverse speed is too high in the actual production, and smaller facula radius should be selected in the reasonable range of technological parameters to obtain the fracture splitting notch with larger ratio of depth to width. The reasonable selection of process parameters should be as follows: output power is from $400 \mathrm{~W}$ to $450 \mathrm{~W}$, transverse speed is $30 \mathrm{~mm} / \mathrm{s}$ and facula radius is $0.06 \mathrm{~mm}$.

\section{Introduction}

Engine connecting rod and crankcase bearing seat are the typical splitting parts, and fracture splitting processing, which is a type of the advanced precision manufacturing process for splitting parts and is also a major breakthrough of the parts manufacturing technology, has developed quickly in recent years. The principle of fracture splitting technology is that the fracture splitting notch is processed at the predetermined position for splitting, then the normal stress perpendicular to the predetermined fracture surface is applied, the parts is separated by brittle fracture along the initial fracture source under the condition that there is almost no plastic deformation, and three dimensional concave and convex surface formed from the fracture splitting process is used for meshing to implement precise localization in three directions between cover and body, as is shown in Fig. 1. Pre-processing a fracture splitting notch is the critical sequence in the fracture splitting processing, and the processing quality of the notch directly affects the subsequent directional splitting procedure or even the quality of final product. Laser grooving has many advantages, such as high-precision, high-efficiency and no breakages, and it has got rapid development and application in pre-processing a fracture splitting notch of connecting rod. At present, pulsed laser is mainly used in pre-processing a fracture splitting notch of connecting rod, and the corresponding researches for pulsed laser grooving already have been done [1-3]. However, instantaneous energy of intense pulsed laser is so high that the process of laser cutting is quite complicated and the cutting quality cannot be controlled easily. Therefore, the exploration of other types of laser grooving is of great significance to improve the quality of fracture splitting notch and even the quality of the subsequent splitting process. Nd:YAG continuous laser has been widely used in the modern industry. As to explore a better and more suitable way to groove by 
laser, the preliminary exploration of continuous laser grooving for fracture splitting was carried out in this study. With the help of finite element analysis software of SYSWELD, which has been widely used in the welding field [4-7] but rarely applied in the other processing fields [8,9], the temperature field of continuous laser grooving of an engine connecting rod was carried out. The shapes and the dimensions of the fracture splitting notch under different parameters were analyzed, which can provide the reference for evaluating and optimizing the technology of continuous laser grooving.

(a)
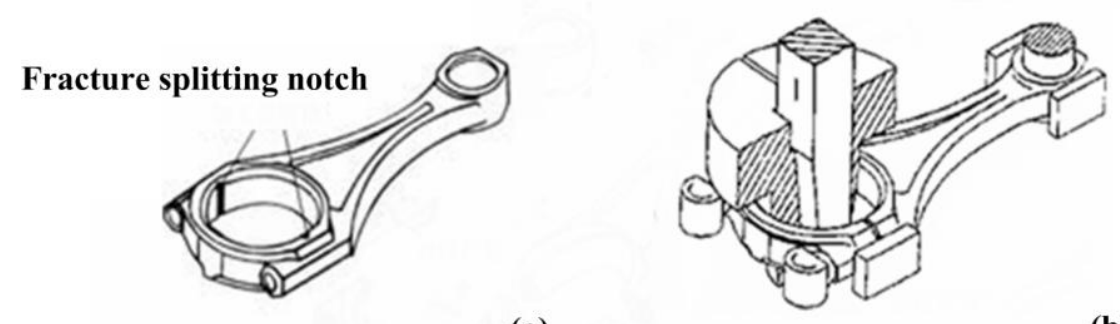

(b)

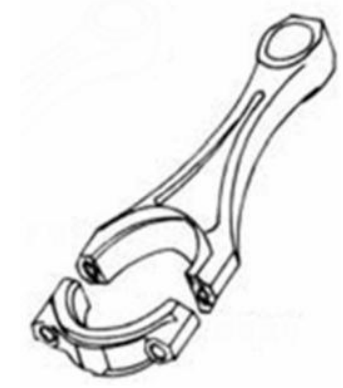

(c)

Fig. 1 Key processes of fracture splitting processing of connecting rod: (a) pre-processing a fracture splitting notch; (b) the splitting process; (c) combined assembly

\section{Theoretical basis}

Basic assumptions. Based on the characteristics of laser grooving for fracture splitting and the essence of laser heat transfer, the following basic assumptions could be considered during constructing the numerical analysis model of continuous laser grooving: (1) the heat affected zone of laser cutting is small, and therefore the continuous laser grooving can be simplified as the heat transfer problem of a moving heat source in a semi-infinite body; (2) the material was isotropic; (3) there was a approximate linear relationship between material thermophysical parameters and temperature in a certain temperature range; (4) the heating effect caused by plasma cloud on the workpiece was not considered; (5) the gravity effect was ignored.

Phase transformation latent heat. Material phase transformation occurs with the absorption or release of energy and such energy can influence the temperature distribution of continuous laser processing. Phase transformation latent heat can be treated by defining the material enthalpy under different temperature. Enthalpy is the integral of the product of specific heat and density for temperature, the expression is shown as follows:

$$
H=\int \rho c(T) d T \text {. }
$$

Where $H$ is the heat enthalpy, $\rho, c, T$ is the density, specific heat and temperature respectively.

When the temperature of a certain node is higher than the phase transformation point, the enthalpy will change. In this way, phase transformation latent heat can be taken into account through the enthalpy change.

\section{Finite element modeling}

Geometric modeling and meshing. The location of the fracture splitting notch of connecting rod is shown in Fig. 1(a). Combined with the previous studies on pulsed laser grooving of an engine connecting rod [10] and the physical process between the laser beam and materials during continuous laser grooving, the connecting rod was simplified to be a cuboid and half of the model was selected for analysis, as is shown in Fig. 2(a). To facilitate meshing regionally, the cube model was divided 
into three parts, $\mathrm{V}_{1}, \mathrm{~V}_{2}$ and $\mathrm{V}_{3}$, and $\mathrm{V}_{1}$ was the laser energy acting region which would develop into the fracture splitting notch and heat affected zone.

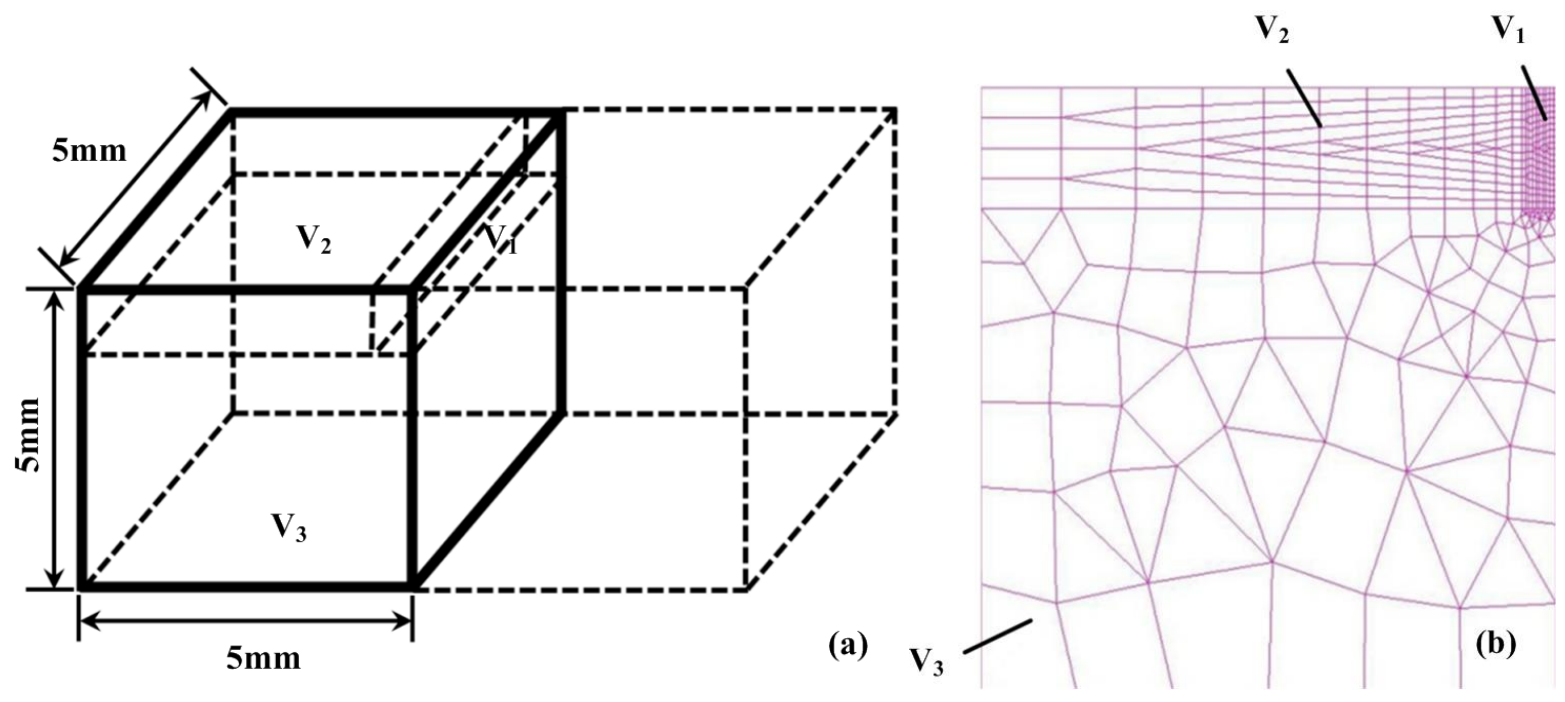

Fig. 2 Geometrical model and mesh generation: (a) geometric model, (b) flat view of mesh

Continuous laser grooving is a process that the local of the workpiece is heated rapidly by the moving laser heat source and quenched subsequently, thus there is a constantly changing temperature gradient at the space points of the laser acting position and their surrounding region. In order to improve the solution efficiency and precision, the length of element in $\mathrm{V}_{1}$ was set to $0.02 \mathrm{~mm}$. While the mesh generation was carried out, the hexahedron element and tetrahedron element was used. For guaranteeing the quality of the mesh, the free meshing was used in $\mathrm{V}_{3}$, the mapped meshing was used in $V_{1}$ and $V_{2}$, and the mesh model is shown in Fig. 2(b).

Technical treatment of material property parameters. Temperature varies rapidly during continuous laser grooving, and therefore the changes of the material thermophysical parameters with temperature should be considered. The engineering database of relevant parameters of steel C70S6 was established by inputting the material thermophysical parameters at typical temperatures. Particularly, parameter values in the unknown range of temperature were determined by the interpolation method and the extrapolation method [11-13].

Initial conditions and boundary conditions. The initial temperature of each node in the model was set to $20^{\circ} \mathrm{C}$. The symmetry plane was regarded as the adiabatic boundary condition. Convection heat transfer exists on the external surface of the workpiece, the high-speed air was used for removing the molten metal on the location of laser beam, and therefore the convective heat-transfer coefficient was applied on the remainder of the surface of the model. In addition, the energy loss caused by radiation was ignored.

Heat source modeling and checking. During the continuous laser grooving for fracture splitting, metal materials is molten by the laser, even parts of the materials is gasified, and the molten region is formed with larger ratio $(\geq 1)$ of depth to width, which shows that the heat flow of laser heat source imposes great influence in the thickness direction of the workpiece. Therefore, 3D gauss conical heat source model was selected in the numerical computation. The essence of 3D gauss conical heat source is the superposition of a series of gauss plane heat sources along the thickness direction of the workpiece, the heat source is distributed within a certain range according to the Gaussian function, and the heat flux distribution radius at each section shows a linear attenuation along the thickness direction, but the heat flux density keeps consistent in the direction of the central line of laser beam ( $\mathrm{z}$ axis). Heat flux distribution function of 3D gauss conical heat source is shown as follows: 


$$
\begin{aligned}
& q(r, z)=\frac{9 \eta P e^{3}}{\pi h\left(e^{3}-1\right)\left(r_{e}^{2}+r_{e} r_{i}+r_{i}^{2}\right)} \exp \left(-\frac{3 r^{2}}{r_{0}^{2}}\right) . \\
& \mathrm{r}_{0}(z)=r_{e}-\left(r_{e}-r_{i}\right)\left(z_{e}-z\right) /\left(z_{e}-z_{i}\right) .
\end{aligned}
$$

Where $r_{\mathrm{e}}$ and $r_{\mathrm{i}}$ is heat flux distribution radius of the upper and lower surfaces of heat source respectively; $z_{\mathrm{e}}$ and $z_{\mathrm{i}}$ is z coordinate of the upper and lower surfaces of heat source respectively; $r_{0}$ is the heat flux distribution radius on the plane of $z$ coordinate; $P$ is laser output power; $\eta$ is effective power coefficient; $h$ is the heat source depth.

Based on the previous studies on the experiment of continuous laser processing grooving of engine crankcase bearing block [14], the heat source was checked to guarantee the authenticity and accuracy of the simulation, and the comparison between the simulation values and experimental results is shown in Fig. 3. Continuous laser grooving for fracture splitting is a physical process which contains laser melting material or even gasifying material, and high-speed airflow blowing molten material. Therefore, the isotherm of material melting-point temperature can be treated as the notch contour line to calculate the dimension of the fracture splitting notch. It can be seen that $3 \mathrm{D}$ gauss conical heat source could simulate the continuous laser grooving effectively.

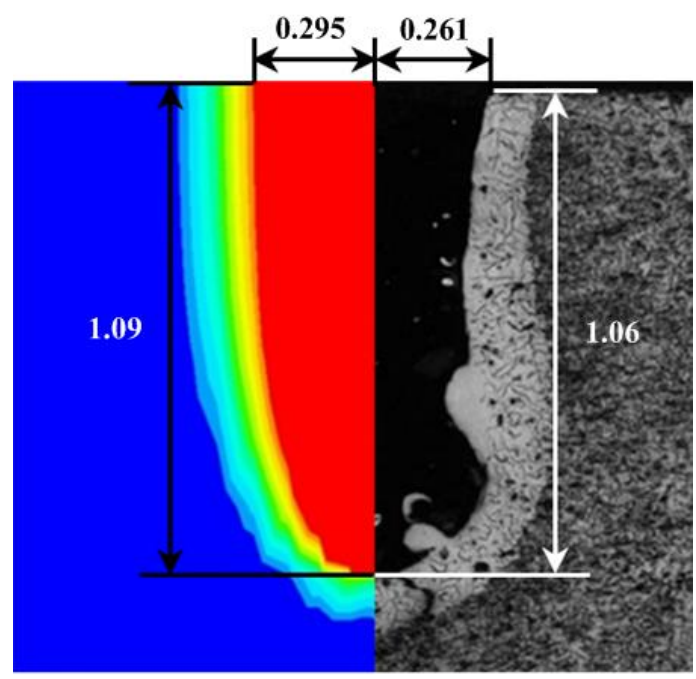

Fig.3 The comparison between the temperature field and experimental results of continuous laser grooving of HT250

It should be remarked that the effective power coefficient was treated as 0.5 when the heat source was checked, the reasons for which are described as follows: (1) There is a part of energy loss in the distance from the injection of laser beam to the irradiation of laser beam on the specimen; (2) there is reflection on the specimen surface; (3) a part of molten material can refreeze after laser cutting, and this part of metal material is not taken into account when the geometry dimension of the notch is calculated, which is the main reason why the value of effective power coefficient in this study is less than the experience value (0.7).

\section{Temperature field of continuous laser grooving}

The selection of process parameters. Process parameters, which affect the temperature field of continuous laser grooving, the dimension and the quality of the fracture splitting notch, mainly include laser output power, facula radius, transverse speed and defocusing amount. Whether the process parameters are selected reasonably would directly affect the quality of the fracture splitting notch and then determine the choice of the laser and the equipment cost. Generally for the different types of connecting rod, the required depth of the notch is $0.45 \mathrm{~mm}-0.7 \mathrm{~mm}$. A few sets of process 
parameters were selected for the numerical simulation of continuous laser grooving by synthesizing the influence of various parameters on the processing quality, as is shown in Table 1.

Table 1 Process parameters of continuous laser grooving

\begin{tabular}{|c|c|c|c|c|}
\hline Set & $\begin{array}{c}\text { Output power } \\
P[\mathrm{~W}]\end{array}$ & $\begin{array}{c}\text { Transverse speed } \\
v[\mathrm{~mm} / \mathrm{s}]\end{array}$ & $\begin{array}{c}\text { Facula radius } \\
r_{\mathrm{e}}[\mathrm{mm}]\end{array}$ & $\begin{array}{c}\text { Defocusing amount } \\
\triangle \mathrm{f}[\mathrm{mm}]\end{array}$ \\
\hline 1 & $450 / 400 / 350 / 300$ & 30 & 0.06 & 0 \\
\hline 2 & 400 & $40 / 35 / 30 / 25$ & 0.06 & 0 \\
\hline 3 & 400 & 30 & $0.10 / 0.06 / 0.04$ & 0 \\
\hline 4 & 400 & 30 & 0.06 & $0.1 / 0 /-0.1$ \\
\hline
\end{tabular}

The quasi-steady state temperature distribution of continuous laser grooving is shown in Fig. 4 under the condition of a certain parameter combinations in table $1\left(P=400 \mathrm{~W}, v=30 \mathrm{~mm} / \mathrm{s}, r_{\mathrm{e}}=0.06 \mathrm{~mm}\right.$, $\Delta \mathrm{f}=0$ ), the dimension of the fracture splitting notch (Notch depth is $0.64 \mathrm{~mm}$ and notch width is $0.27 \mathrm{~mm}$.) was obtained by measuring the simulation results, and the specific dimension of the notch met the requirement in practical production.

Temperature/ ${ }^{\circ} \mathrm{C}$

Temperature $/{ }^{\circ} \mathrm{C}$
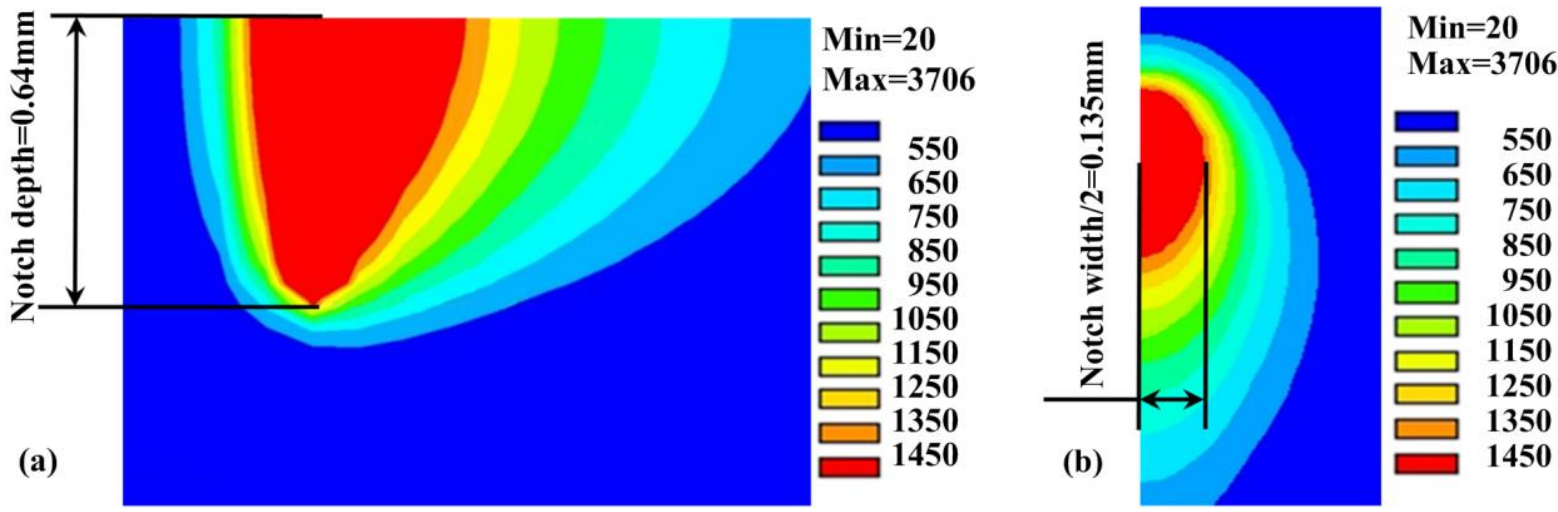

Fig.4 The dimension of fracture splitting notch: (a) notch depth, (b) notch width

The influence of output power and transverse speed on the dimension of the fracture splitting notch. Output power directly affects the energy density of heat source and reflects the ability of laser melting material; moreover, transverse speed determines the time of continuous laser irradiating the workpiece. Therefore, the heat input to the processing workpiece is determined by output power and transverse speed. According to the set 1 and 2 of process parameters in Table 1, the temperature field simulation of continuous laser cutting steel C70S6 was carried out. The dimension change curves of the fracture splitting notch with output power and transverse speed are shown in Fig. 5.

Output power has great influence on notch depth, and notch depth is almost proportional to output power, as is shown in Fig. 5(a). When output power increases from $300 \mathrm{~W}$ to $450 \mathrm{~W}$, notch depth increases from $0.53 \mathrm{~mm}$ to $0.72 \mathrm{~mm}$. But notch width changes slightly with output power, when output power increases from $300 \mathrm{~W}$ to $450 \mathrm{~W}$, notch width increases slightly from $0.25 \mathrm{~mm}$ to $0.3 \mathrm{~mm}$. Transverse speed has greater effect on notch depth and notch width, as is shown in Fig. 5(b). Notch depth and notch width decreases gradually as transverse speed rises. When transverse speed increases from $40 \mathrm{~mm} / \mathrm{s}$ to $25 \mathrm{~mm} / \mathrm{s}$, notch depth decreases from $0.66 \mathrm{~mm}$ to $0.58 \mathrm{~mm}$, and notch width decreases from $0.32 \mathrm{~mm}$ to $0.23 \mathrm{~mm}$. Transverse speed can affect the production efficiency; although increasing transverse speed can improve the cutting efficiency and get a relatively narrow notch, the difficulty in eliminating the molten metal can be increased and notch depth can decrease, the splitting force could be increased in the subsequent splitting process and then the processing quality of fracture splitting processing could be influenced. Therefore, it is not recommended that transverse speed is too high in the actual process of continuous laser grooving. 

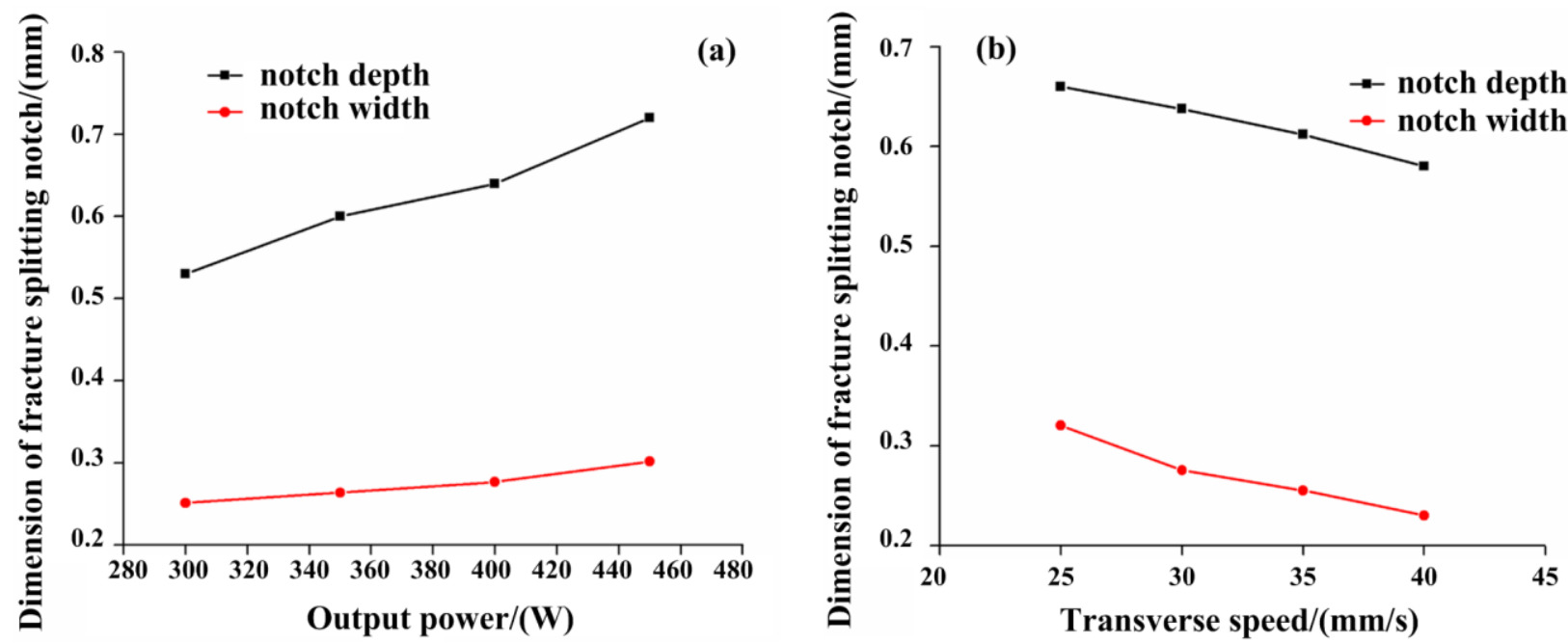

Fig.5 The influence of output power and transverse speed on the dimension of fracture splitting notch:

(a) the dimension of fracture splitting notch-output power, (b) the dimension of fracture splitting notch-transverse speed

The influence of facula radius and defocusing amount on the dimension of the fracture splitting notch. Facula radius, which determines the energy intensity of laser beam, represents the interaction region between laser beam and workpiece. Defocusing amount is the relative position between the focus of laser beam and the processed surface of the workpiece. When defocusing amount is changed, the facula radius of laser beam acting on the processed surface and the effective action depth would change accordingly, which would cause the changes of the geometry dimension of the fracture splitting notch. For the set 3 and 4 of process parameters in Table 1, the effects of facula radius and defocusing amount on the dimension of the fracture splitting notch during continuous laser cutting steel C70S6 are shown in Fig. 6.
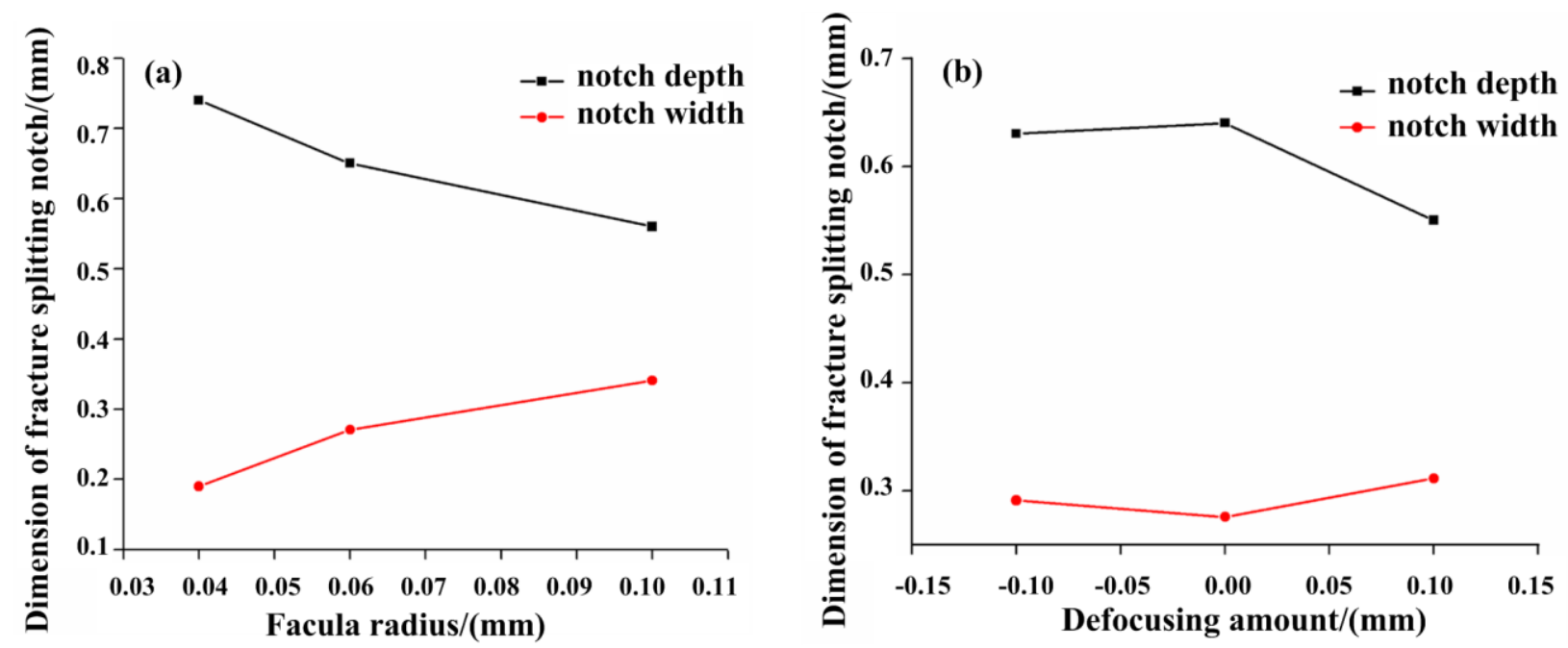

Fig.6 The influence of facula radius and defocusing amount on the dimension of fracture splitting notch: (a) dimension of fracture splitting notch-facula radius, (b) dimension of fracture splitting notch-defocusing amount

Fig. 6(a) shows that facula radius has greater influence on notch depth and notch width. Facula radius is proportional to notch width, but it is inversely proportional to notch depth. When facula radius increases from $0.04 \mathrm{~mm}$ to $0.10 \mathrm{~mm}$, notch depth decreases from $0.74 \mathrm{~mm}$ to $0.56 \mathrm{~mm}$, and notch width increases from $0.19 \mathrm{~mm}$ to $0.34 \mathrm{~mm}$. Therefore, the smaller facula radius should be selected in the reasonable range of process parameters to obtain a fracture splitting notch with larger ratio of 
depth to width. It can be seen from the Fig. 6(b) that when defocusing amount is negative values, notch depth keeps consistent with the corresponding depth when defocusing amount is zero basically. However, when defocusing amount is positive values, notch depth decreases obviously. When defocusing amount is zero, notch width is the minimum value, and the width can increase regardless of the increase or decrease of defocusing amount, which accords with the actual rule of laser cutting. In the actual production of continuous laser processing of notches, defocusing amount should be set to zero or a very small negative value.

Synthesizing the influence of the process parameters on the geometry dimension of the fracture splitting notch, process parameters should be selected reasonably as follows: output power is from $400 \mathrm{~W}$ to $450 \mathrm{~W}$, cutting speed is $30 \mathrm{~mm} / \mathrm{s}$ and facula radius is $0.06 \mathrm{~mm}$.

\section{Summary}

According to the actual physical process between the laser beam and materials during laser processing, the finite element analysis model was built for continuous laser grooving for fracture splitting, and the key problems during modeling, such as the establishing and checking of laser heat source model, the nonlinearity of material thermophysical parameters and the phase transformation latent heat, were discussed and solved.

For the continuous laser grooving for fracture splitting of a C70S6 connecting rod, the notch dimension obtained from the simulation results met the production requirement; the influence rules of output power, transverse speed, facula radius and defocusing amount on the geometric parameters of fracture splitting notch were analyzed. Notch depth and notch width was positively correlated with laser output power but negatively correlated with transverse speed. Facula radius was negatively correlated with notch depth but positively correlated with notch width. The reasonable selection of process parameters should be as follows: output power is from $400 \mathrm{~W}$ to $450 \mathrm{~W}$, transverse speed is $30 \mathrm{~mm} / \mathrm{s}$ and facula radius is $0.06 \mathrm{~mm}$.

\section{Acknowledgements}

This work was financially supported by National Science and Technology Major Project of China (No.2013ZX04002-021).

\section{References}

[1] S.Q. Kou, J.W. Wang, X.X. Jian and Q.F. Zheng: Opt. Precision Eng. Vol. 18 (2012), p. 1340-1346

[2] S.Q. Kou, J.W. Wang and Y. Gao: Laser. Eng. Vol., 20 (2010), p. 381-395

[3] S.Q. Kou, J.W. Wang, Q.F. Zheng and S.H. Yang: Opt. Precision Eng. Vol. 19 (2011), p. 773-779

[4] U. Reisgen, S. Olschok, M. Mavany and S. Jakobs: Physics Procedia Vol. 39 (2012), p. 75-83

[5] J. Winczek: J. Mater. Process. Technol. Vol. 212 (2012), p. 1080-1088

[6] R. Prakash and R. Gangradey: Fusion Eng. Des. Vol. 88 (2013), p. 2581-2584

[7] J.R. Chukkan, M. Vasudevan and S. Muthukumaran: J. Mater. Process. Technol. Vol. 219 (2015), p. $48-59$

[8] E. Feulvarch, M. Fontaine and J.M. Bergheau: Finite Elem. Anal. Des. Vol. 75 (2013), p. 62-70

[9] D.G. Ahn, K.W. Byun and M.C. Kang: J. Mater. Sci. Technol. Vol. 26 (2010), p. 362-366

[10]S.Q. Kou, J.W. Wang and Q.F. Zheng: Laser. Eng. Vol. 21 (2011), p. 115-127 
[11]I. Chen and S. Lee: J. Appl. Phys. Vol. 54 (1983), p. 1062-1066

[12]D.J. Sanders: Appl. Opt. Vol. 23 (1984), p. 30-35

[13] A.F.H. Kaplan: J. Appl. Phys. Vol. 79 (1996), p. 2198-2208

[14]G. Yu: Research on fracture splitting processing technology and equipment of engine crankcase bearing block (Jilin University, Changchun 2012). 